\title{
A Mathematical Approach for Load Balancing in 4G LTE Networks through Wi-Fi APS and Smart Algorithm
}

\author{
Hadeel Khairy A.Aldaly, Osama Mohamedahmed Suliman ,AmmarAbdelrahimMuddathir \\ Ali.M.A.Ibrahim,Sami Abbas Nagar
}

\begin{abstract}
-.there is a rapid growth in cellular networks due to the huge turnout for it, but all this happening without the current generations being fully utilized in terms of spectrum. Due to the nature of the cellular system where areas are divided into cells that have limited capacity, some areas might have larger populations causing the full utilization (congestion) of the cell whereas other areas might be serving a far less user base leading to the underutilization of the cell meaning that the overall system isn't utilized properly. In order to solve this problem load balancing has been proposed with different approaches that ranged from tinkering with the cell reselection parameters or the cell's shape and coverage. However these methods have failed to catch on thus far for a number of reasons and load balancing is still currently done manually using radio engineers. In our research we explore a mathematical model for load balancing in 4G LTE networks. We have proposed the addition of Wi-Fi APs as well as a smart algorithm in order to carry out the load balancing procedure.
\end{abstract}

Key word: 4G,load balancing, offloading, Wi-Fi Aps, Algorithm

\section{Introduction}

The congestion can cause some serving cells to be overloaded with heavy traffic whilst other cells maybe serving less users with less demand and are thus lightly loaded causing the cellular system to suffer from unbalanced loads that degrades its overall performance and utilization.

The objective of this research is to develop and validate a mathematical model for load balancing in $4 \mathrm{G}$ LTE networks through Wi-Fi APs and smart algorithm to address the congestion problem without altering the system bandwidth by working on the three main matrices (Delay, Throughput and Packet Loss).

\section{Related work}

The effects of congestion on a network depend on many factors such as the degree of congestion and the type of application. The effects can range from webpages loading much slower or some applications failing completely such as an online transaction timing out [1].

As previous solutions some researchers used the concept of Load Balancing between cells in the same cluster.

The main concept behind load balancing is the equalization of loads experienced by cells carried out by transferring loads from heavily loaded cells to other cells that are less loaded. But it has some drawbacks.

A lot of methods have been suggested to solve the load balancing problem, with most of these methods designed based on the specification (MAC and physical layer) of a particular cellular system, making them applicable to only that particular generation. Thus newer generations such as LTE-A, LTE, 5G are not supported by these solutions [1].

[2-6] have offered many load balancing strategies based on this idea however it should be noted that borrowing of channels doesn't work in LTE and more advanced networks such as LTE-A that utilize OFDMA due to intra-channel interference $[1,7]$

Controlling this phenomena is beyond the operator's control thus operators invest heavily in networks in order to avoid congestion. Operators may try to install more cells sites (for example utilize micro-cells in LTE networks [2], use a more efficient technology or offload data onto other networks such as Wi-Fi [1].

This paper is organized as follows Abstract Section I is an introduction and a brief information about the whole research Section II contains the related work. Section III is about the methodology of Modeling of WiFi Assisted Load Balancing using Smart Algorithm after the explanation of both the study of 4G LTE network behavior and the load balancing main concept. Section IV is the results and discussion of what have been done. Section $\mathrm{V}$ is the conclusion that shows the results that have been observed. While section VI represents the recommendations and the future work.

\section{Methodology}


The problem can be solved using algorithms to help balance the loads on the cells and improve the system utilization.

\section{A. Studying the behaviour of $4 \mathrm{G}$ LTE Network}

The first step of the work had been done by experimenting the 4G LTE network in the real world in order to develop an understanding of how congestion occurs on 4G LTE networks.

Calculation of delay, packet loss and throughput

Throughput $=\frac{\text { received data } * 8}{\text { Data transmission period }}$

Average delay $=\frac{\text { Total delay }}{\text { Count }}$

Delay=receiving time[i]- sending time [i]

Total delay $=$ Total delay + delay[i]

Packet loss $=$ GeneratedPackets - ReceivedPackets

\section{B. Mathematical Model}

\section{i. Load Balancing in $4 G$ LTE networks}

After the 4G LTE network had been studied a mathematical model elaborated based on the work of (II.A) that will be served as an input to the proposed solution.

Whenever a cell experiences a load above a set threshold (i.e. the congestion threshold) it should calculate the share of traffic it should transfer to other non-congested cells in order to satisfy the best QoS (quality of service) for its users.

a. cluster model:

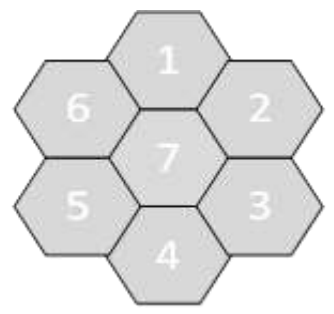

Figure 1. Cluster model that was used in the load balancing experiment

Let:

- $\quad C$ denote the number of non-congested cells;

- $T$ and $B$ denote the traffic and blocking probability of the congested cell;

- $\quad T c$ and $B c$ (where $c=1,2,3, \ldots, C$ ) denote the blocking probability and the current traffic of the non-congested cells

- $T_{f}$ represents the system threshold which is determined by the blocking probability

- $\Delta T$ denote the amount of traffic that is expected to be transferred on load balancing by the congested cell

- $\quad P c$ (where $c=1,2,3, \ldots C$ ) denotes the percentage of traffic that is distributed to each noncongested cell

- $\Delta T c$ denote the amount of traffic actually transferred to each non-congested cell.

For each non-congested cell $\Delta T c$ equals:

$$
\Delta \mathrm{T}_{\mathrm{c}}=\Delta \mathrm{T}-\mathrm{P}_{\mathrm{c}}-\left(1-\mathrm{B}_{\mathrm{c}}\right) \quad(\mathrm{c}=1,2, \ldots, \mathrm{C})
$$

For the congested cell the transferred traffic is:

$\mathrm{T}-\mathrm{T}_{\mathrm{f}}=\sum_{\mathrm{c}=1}^{\mathrm{C}} \Delta \mathrm{T}_{\mathrm{c}}=\sum_{\mathrm{c}=1}^{\mathrm{C}} \Delta \mathrm{T} \cdot \mathrm{P}_{\mathrm{c}} \cdot\left(1-\mathrm{B}_{\mathrm{c}}\right)=\Delta \mathrm{T} \cdot \sum_{\mathrm{c}=1}^{\mathrm{C}} \mathrm{P}_{\mathrm{c}} \cdot\left(1-\mathrm{B}_{\mathrm{c}}\right)$

Therefore $\Delta T$ can be calculated as follows:

$\Delta \mathrm{T}=\frac{\mathrm{T}-\mathrm{T}_{\mathrm{f}}}{\sum_{\mathrm{c}=1}^{\mathrm{c}} \mathrm{P}_{\mathrm{c}}-\left(1-\mathrm{B}_{\mathrm{c}}\right)}$

Using equation 3.1 and 3.3 we can calculate $\Delta T c$ :

$\Delta T_{\mathrm{c}}=\frac{\mathrm{T}-\mathrm{T}_{\mathrm{f}}}{\sum_{\mathrm{c}=1}^{\mathrm{c}} \mathrm{P}_{\mathrm{c}}-\left(1-\mathrm{B}_{\mathrm{c}}\right)}-\mathrm{P}_{\mathrm{c}} \cdot\left(1-\mathrm{B}_{\mathrm{c}}\right) \quad(\mathrm{c}=1,2, \ldots, \mathrm{C})$

Erlang B formula is followed by B (\& Bc) and T (\& Tc) meaning for each cell:

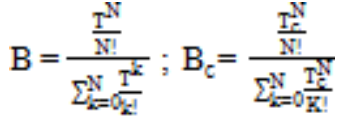


Where $\mathrm{N}$ denotes the number of channels utilized by the system.

Using this formula we can calculate $T_{f}$ by substituting $B$ with $B_{f}$ that denotes the blocking probability of the system.

In order to distribute traffic evenly between cells the parameter Pc has to be properly set:

$$
\mathrm{P}_{\mathrm{c}}=\frac{1 / T_{c}}{\sum_{c=1}^{\mathrm{C}} 1 / T_{c}}
$$

\section{ii. $\quad$ Modeling of Wi-Fi Assisted Load Balancing using Smart Algorithm}

This part explores the usage of Wi-Fi along with a smart algorithm in order to carry out load balancing.

The model has been shown to perform well in scenarios involving multiple congested cells as well an overall good usage of available resources.

Wi-Fi APs of 802.11n standard will be installed in each cell to facilitate the transfer of traffic from one cell to another where the blocking probability was $2 \%$ (equivalent to 17.5 Erlang) for a cell to be fully utilized .Whenever a cell experiences congestion it will carry out calculations in order to determine how much traffic it should transfer as well as which cell it should select to load balance to.

The load balancing procedure will take place when a cell has exceeded the congestion threshold and then proceeds to calculate the utilization and blocking probabilities for each cell to other cells in order to determine the most suitable cell to be the controller of the load balancing procedure (the cell with the least amount of traffic) and then proceeds to carry out load balancing related operations.

\section{a. Cluster model:}

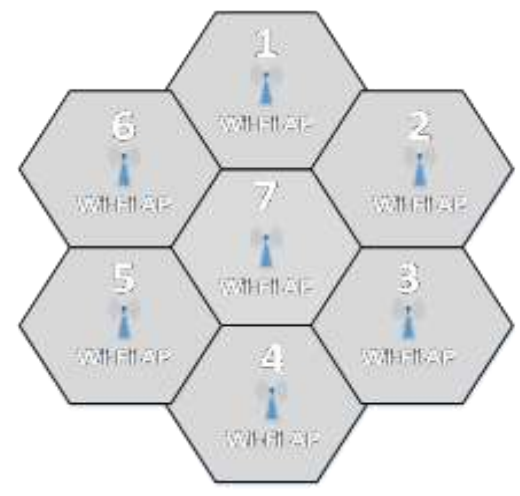

Figure 2. Cluster model with Wi-Fi assisted

i. Traffic values assumption for three scenarios:

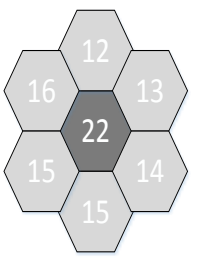

Scenario 1

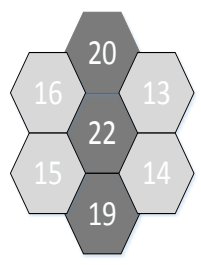

Scenario 2

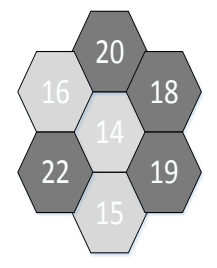

Scenario 3
Figure 3. traffic values

\section{Results and Discussions}

The data of 4G LTE network experiment was captured using Wireshark and then the three matrices have been calculated after it was converted into an excel sheet as shown in equations (1-3).

Fig 4 below shows the new traffic values in Erlang and the corresponding blocking probability for each cell after load balancing. 


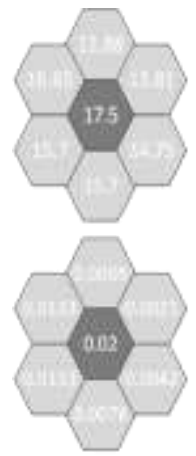

Scenario 1

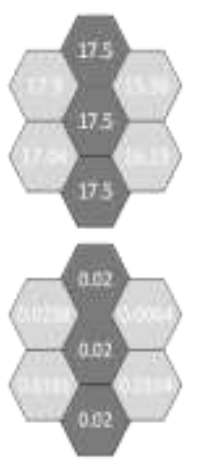

Scenario 2

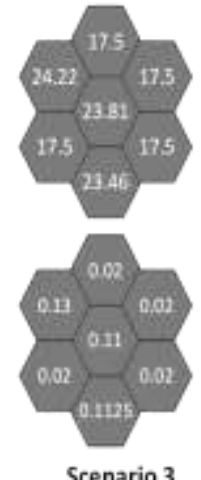

Scenario 3
Figure 4. Result value

Figure below shows the new traffic values

in Erlang and the corresponding blocking probability for each cell when using the Wi-Fi assisted load balancing using smart algorithm
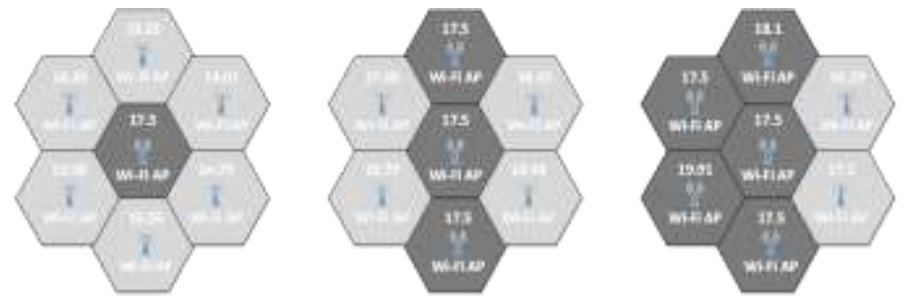

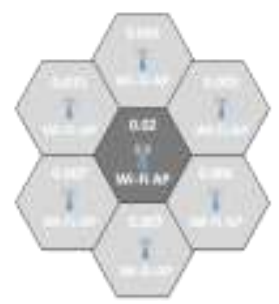

Scenario 1

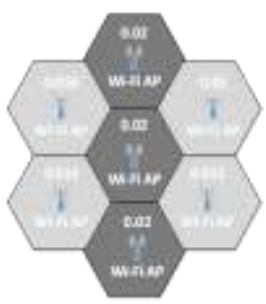

Scenario 2

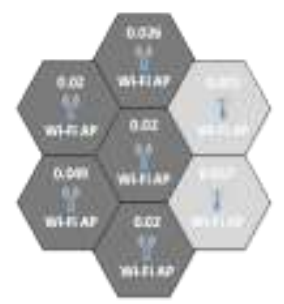

Scenario 3
Figure 5. Result value

As shown in fig 5 above, the proposed solution outperforms the previous mathematical model. It can be seen that the system attempts to utilize the available resources better as well as being able to handle better scenarios involving more than one congested

\section{Conclusion}

This paper contributed an approach for load balancing for the congested cells within the 4G LTE networks, which had been done by a mathematical modeling of load balancing in 4G LTE networks and were proposed the addition of Wi-Fi APs as well as a smart algorithm to the existing network, where this model must have to perform well in utilizing the system resources.

As recommendations of this study are to investigate the performance of this solution over data traffic as well as voice traffic, also there must be some consideration have to be taken into account such as the effect of noise and interference which are a real concern for cellular networks, and also for a better performance over long distances the usage of 802.11ah instead 802.11n seems to better fit due to a number of benefits due to the low power and long range offered by 802.11 ah

\section{Acknowledgment}

Our utmost gratitude goes to Almighty God for giving us an opportunity to this challenging and wonderful work to pursue an undergraduate program in University Of Medical Sciences And Technology (UMST). Our sincere gratitude goes to Dr.Sami A/Nagar, PhD, Senior Member, IEEE for his effective advices and guiding,by giving useful comment and professional suggestions,starting from the research proposal,initiation of the thesis and to its completion. Our gratefulness to Tr.Ali Maher for supervising us to achieve the best outcomes of our project.Also we would like to thank our families for their patience, encouragement, moral and financial support,also to help us to overcome various challenges that we have passed through during our journey.

\section{References}

1. Aliu, O.G., et al., A survey of self organisation in future cellular networks. IEEE Communications Surveys \& Tutorials, 2013. 15(1): p. 336-361.

2. Das, S., et al. An efficient distributed channel management algorithm for cellular mobile networks. in Universal Personal Communications Record, 1997. Conference Record., 1997 IEEE 6th International Conference on. 1997. IEEE.

3. Das, S.K., S.K. Sen, and R. Jayaram. A structured channel borrowing scheme for dynamic load balancing in cellular 
networks. in Distributed Computing Systems, 1997.

Proceedings of the 17th International Conference on. 1997. IEEE.

4. Kim, S. and P.K. Varshney. Adaptive load balancing with preemption for multimedia cellular networks. in Wireless Communications and Networking, 2003. WCNC 2003. 2003 IEEE. 2003. IEEE.

5. Kuboye, B., et al. Development of a Prioritized Scheduling Algorithm for Congestion Management in Long Term Evolution. in Proceedings of the World Congress on Engineering and Computer Science. 2016.

6. Mitra, S. and S. DasBit. On location tracking and load balancing in cellular mobile environment-a probabilistic approach. in Electrical and Computer Engineering, 2008. ICECE 2008. International Conference on. 2008. IEEE.

7. Mishra, S. and N. Mathur, Load Balancing Optimization in LTE/LTE-A Cellular Networks: A Review. arXiv preprint arXiv:1412.7273, 2014

About Author (s):

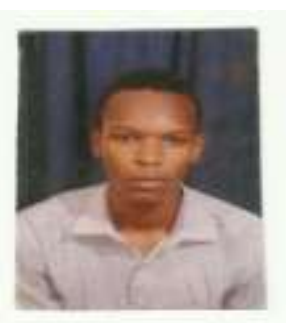

\section{Osama mohamedahmed}

Currently is a graduate Electronic Engineering student in University of Medical Sciences and Technology (UMST),Faculty of Engineering (Electronics-Communication).

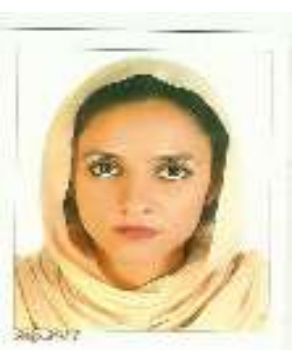

Hadeel Khairy A.Aldaly Currently is a graduate Electronic Engineering student in University of Medical Sciences and Technology (UMST),Faculty of Engineering (ElectronicsCommunication) 